\title{
UTILIZAÇÃO DE ISOTRETINOÍNA ORAL NO TRATAMENTO DA ALOPECIA FIBROSANTE FRONTAL: SÉRIE DE CASOS
}

\author{
USE OF ORAL ISOTRETINOIN IN THE TREATMENT OF FRONTAL \\ FIBROSANT ALOPECIA: CASE SERIES
}

\author{
Neuza Caroline Suassuna Araújo ${ }^{1}$ \\ Lívio e Vasconcelos do Egypto ${ }^{2}$
}

RESUMO: Introdução: A alopecia fibrosante frontal (AFF) é considerada uma alopecia linfocítica primária de caráter crônico, sendo descrita também como um tipo de alopecia cicatricial. Clinicamente, é comum o paciente acometido por tal patologia apresentar recessão frontotemporal da linha do cabelo, frequentemente acompanhada por perda de sobrancelha. Embora tenha um padrão clínico bem característico, ainda não se sabe ao certo sua patogênese, existindo diversas hipóteses para tal distúrbio patológico. O prognóstico para casos dessa natureza é fundamentado pelo estágio em que se encontra. Destaque-se, que não há, ainda, um tratamento específico para a AFF. Objetivo: Nesta direção, a presente pesquisa objetivou, em linhas gerais, avaliar os efeitos terapêuticos da utilização da isotretinoína oral em pacientes que foram diagnosticadas com alopecia fibrosante frontal. Método: Para o estudo ora apresentado, realizou-se uma descrição de uma série de casos, caracterizando-se, pois, como um estudo observacional de caráter descritivo, com abordagem de natureza qualitativa. As informações foram obtidas por meio de entrevistas e de coleta de dados dos prontuários dos pacientes, com autorização expressa, por escrito, mediante assinatura do Termo de Consentimento Livre e Esclarecido (TCLE), devidamente submetido ao Comitê de Ética em Pesquisa do Centro Universitário de Patos (UNIFIP). Resultados: Entende-se que este estudo poderá contribuir para melhor conhecimento acerca dos efeitos terapêuticos no curso clínico da utilização da terapia medicamentosa com isotretinoína oral em pacientes diagnosticados com AFF, com vistas a proporcionar auxílio aos profissionais especializados em relação à conduta medicamentosa que apresente resposta favorável à evolução clínica do paciente. Conclusão: $O$ tratamento com isotretinoína oral e se mostrou eficaz, pois ocorreu a estabilização

\footnotetext{
${ }^{1}$ Graduanda do curso de bacharelado em Medicina no Centro Universitário de Patos - UNIFIP, PatosPB.neuzaaraujo@med.fiponline.edu.br.

2 Médico Dermatologista pela Universitade. Docente do Curso de Medicina do Centro Universitário de

Patos - UNIFIP, Patos-PB. livioegypto@hotmail.com.
} 
da alopecia. A perda capilar gera impacto na feminilidade dessas mulheres, sendo comum a relação da alopecia com baixa autoestima.

Palavras chave: Alopecia fibrosante frontal; Isotretinoína; Efeitos terapêuticos.

ABSTRACT: Introduction: Frontal fibrosing alopecia (AFF) it is considered a primary lymphocytic alopecia of a chronic character, and it is also described as a type of cicatricial alopecia. Clinically, it is common to have frontotemporal recession of the hairline, often accompanied by eyebrow loss. Although it has a very characteristic clinical pattern, its pathogenesis is not yet known, with several hypotheses. The prognosis of this pathology is influenced by the stage it is in. In addition, it does not have a specific treatment for AFF. Objective: The objective was to evaluate the therapeutic effects of using oral isotretinoin in patients who were diagnosed with frontal fibrosing alopecia. Methods: In this study, a description of a series of cases was performed. This research has the characteristic of being an observational study of a descriptive character and presents a qualitative approach. The information will be obtained through interviews and data collection from the patients' medical records, this can only be done after written authorization by signing the informed consent form (ICF) and the consent of the Research Ethics Committee of the University Center of Patos (UNIFIP). Results: This study may contribute to better know the therapeutic effects in the clinical course of the use of drug therapy with oral isotretinoin in patients diagnosed with AFF, thus providing assistance to professionals in relation to drug management that presents a favorable response to the clinical evolution of the patient. Conclusion: The treatment with oral isotretinoin has demonstrated certain efficacy, resulting in the stabilization of the alopecia. The capillary loss generates and impact of the women's femininity, which indicates the relation between alopecia and low esteem.

Keywords: Frontal fibrosing alopecia; Isotretinoin; Therapeutic effects. 


\section{INTRODUÇÃO}

A alopecia fibrosante frontal (AFF) foi descrita primordialmente em 1994 por Kossard e é considerada uma alopecia cicatricial primária. O padrão clínico da AFF é caracterizado por recessão progressiva e simétrica da linha frontotemporal do couro cabeludo. Em grande parcela da população que tem o diagnóstico de AFF, o quadro clínico pode ser acompanhado por outras características, tais como: perda dos pelos da sobrancelha, perda de pelos corporais e surgimento de pápulas faciais (RAKOWSKA et al., 2017).

Quanto à epidemiologia foi relatada inicialmente como uma patologia que acomete apenas em mulheres no período pós-menopausa, atualmente foram surgindo novos relatos, nos quais a AFF passou a acometer também mulheres em idade fértil e homens. Contudo, essa patologia ainda é descrita na literatura tendo maior distribuição em mulheres em uma faixa etária em torno dos 66 anos (SMIDARLE; SEIDL; SILVA, 2010).

A patogênese da AFF ainda não foi elucidada, no entanto existem várias teorias sobre fatores que podem ser desencadeantes, como a ação hormonal, a inflamação neurogênica, o tabagismo, os filtros ultravioletas (UV) e ingredientes de produtos para rosto sem enxágue, além dessas, pressupõe também uma base genética, pois a AFF pode se mostrar presente em membros de uma mesma família (LORIZZO; TOSTI, 2019). É importante ressaltar que mesmo havendo uma diversidade de hipóteses nenhuma delas explica ao certo como a patologia se desenvolve.

Por apresentar um quadro clínico bem característico, o diagnóstico torna-se fácil de ser estabelecido. No entanto, em estágios iniciais pode apresentar uma dificuldade em distinguir a AAF de outros diagnósticos (ESTABAN-LUCÍA; MOLINARUIZ; REQUENA, 2017).

Dessa forma, para confirmar o diagnóstico pode ser feito uma análise histopatológica do couro cabeludo da região acometida, que poderá evidenciar a 
alopecia cicatricial com presença de folículos pilosos fibrosados e infiltração linfocítica circundando o folículo piloso com presença de folículos pilosos fibrosados, além da infiltração linfocítica (MULINARI-BRENNER et al., 2007).

$O$ tratamento da AFF ainda é alvo de pesquisas, pois ainda não existe um tratamento validado ou aprovado. A terapia medicamentosa é prolongada, pois a AFF apresenta caráter crônico. A falta de ensaios clínicos randomizados faz com que não se saiba ao certo a eficácia dos tratamentos disponíveis (LORIZZO; TOSTI, 2019).

A alopecia fibrosante frontal é uma comorbidade de etiologia pouco conhecida, muitas das vezes é passada despercebida, sendo diagnosticada como outra patologia. Mesmo já existindo pesquisas sobre o assunto atualmente ainda não existe uma terapia medicamentosa elucidada. Assim sendo, o trabalho descreverá uma série de casos mostrando os possíveis efeitos da utilização da isotretinoína nessa patologia. Dessa forma, tem-se como objetivo auxiliar os profissionais a uma conduta medicamentosa que apresente resposta favorável a evolução clínica do paciente.

\section{MATERIAL E MÉTODOS}

O relato de caso é definido como uma descrição detalhada de casos clínicos, contendo características importantes sobre sinais, sintomas e outras características do paciente e relatando os procedimentos terapêuticos utilizados, bem como o desenlace do caso. É caracterizada como um estudo observacional, prospectivo, de caráter descritivo e de abordagem qualitativa. Relatos de caso e séries de casos constituem o método de eleição pelo qual profissionais da saúde descrevem cientificamente sua experiência contribuindo com isso para o progresso do conhecimento médico (PARENTE; OLIVEIRA; CELESTE, 2010; KIENLE; KIENE, 2011).

Os relatos de casos, ou mesmo séria de casos, têm um valor central no descobrimento de novas doenças, tratamentos, efeitos inesperados, efeitos 
colaterais e para o ensino. Eles constituem importantes ferramentas do progresso da medicina; praticamente todas as inovações terapêuticas se iniciam com o relato de poucas evoluções clínicas de pacientes, podendo fornecer informações fundamentais para a melhor decisão na conduta clínica, além de disponibilizar dados clínicos importantes para a prática clínica. Devido a sua importância, espera-se que os relatos de caso tenham ótima qualidade na sua preparação e exposição, com conteúdo e sequência apropriados, tal como os estudos clínicos (PARENTE; OLIVEIRA; CELESTE, 2010; KIENLE; KIENE, 2011).

Para a pesquisa aqui descrita, o estudo dos casos foi realizado na Clínica Neurederm Serviços Médicos LTDA e no Centro de Especialidades Frei Damião, ambos localizados na cidade de Patos-PB, no período de fevereiro a abril de 2020 . As pacientes escolhidas como amostra para o caso são pardas, encontram-se na faixa etária entre 40 e 60 anos, foram diagnosticadas com alopecia fibrosante frontal e tratadas com isotretinoína oral. Após a aprovação pelo Comitê de Ética e Pesquisa (CEP) do Centro Universitário de Patos - UNIFIP, com número do parecer do CAAE 39770120.6.0000.5181, as pacientes foram contatadas e assim foi realizado esclarecimentos sobre os objetivos da pesquisa com posterior assinatura do Termo de Consentimento Livre e Esclarecido (TCLE) e o Termo de Assentimento. Com isso, os trâmites que envolvem a pesquisa foram realizados condizentes às normas da Resolução número 466/2012 e 510/16 do Conselho Nacional de Saúde Pesquisa em Ciências Humanas e Sociais.

As informações foram coletadas a partir de prontuários eletrônicos e por meio de entrevistas, com ênfase na anamnese, investigação clínica, localização da alopecia, forma clínica, exames complementares laboratoriais e histopatológicos, além do tratamento realizado e a evolução clinica das pacientes. Os dados foram digitalizados e sistematizados no programa Microsoft Word 2016®, com posterior análise qualitativa e interpretação crítica. Por fim, os estudos utilizados para a discussão deste trabalho foram extraídos das seguintes bases de dados Biblioteca Virtual em Saúde (BVS), Medical Publisher (PUBMED), IBECS e MEDLINE, obtidos por meio dos descritores "frontal fibrosing alopecia" AND "isotretinoin" AND "therapy". 


\section{RELATO DE CASO}

\section{Paciente 1}

A Paciente A, do gênero feminino, 40 anos, parda, casada, com segundo grau completo, atua como auxiliar de saúde bucal, residente e procedente da cidade de Imaculada-PB. A paciente procurou o serviço de dermatologia da Clínica Neurederm, em 2017, queixando-se de alopecia, principalmente na região frontal, há cerca de um ano e seis meses. Relatou que de início apresentou madarose, há aproximadamente oito anos, e, dois anos mais depois, a alopecia se instalou. Negou demais queixas, comorbidades, alergia a medicações, etilismo e tabagismo.

Baseado nas manifestações e características clínicas da alopecia foi levantada a hipótese diagnóstica de alopecia fibrosante frontal. Diante disso foi solicitado uma biopsia, cuja macroscopia revelou fragmento irregular de tecido com 0,4×0,2cm, pardo claro, firme e elástico; já na microscopia, observou-se cortes histológicos seriados, nas quais exibiram, na derme, estruturas foliculares associadas à fibroplasia lamelar concêntrica com infiltrado inflamatório linfoplasmocitário periférico.

O diagnóstico foi descrito como um quadro histopatológico que favorece o quadro clínico de Alopecia Cicatricial Centrífuga Central. Além da biopsia, foram solicitados os seguintes exames laboratoriais: hemograma, TSH, T4 livre, T3, antireoglobulina, antiperoxidade, FAN, anticorpo antifosfolípede, função hepática (TGO, TGP, GGt, Fosfatase Alcalina), ureia, creatinina e VHS, contudo todos os resultados estavam dentro dos parâmetros normais.

Após confirmação do diagnóstico, iniciou-se o tratamento (04/2017) composto por finasterida $(2 \mathrm{mg} / \mathrm{dia})$ + fluoxetina $(20 \mathrm{mg} / \mathrm{dia})$ + prednisona (20mg/dia), com duração de aproximadamente três meses (até 07/17), o qual foi interrompido por conta própria. Em agosto de 2017, reiniciou-se a terapêutica com minoxidil spray $(5 \%)+$ [hidrato de calcipotriol + dipropionato de betametasona (pomada)] 
(50mcg/g+0,5mg), à noite. Em novembro de 2017, a referida paciente retornou ao ambulatório e informou que estava há um mês sem uso da medicação, pelo que foi observado um aumento da área de calvície.

Nessa mesma ocasião, foi repetido o último esquema terapêutico e adicionada a finasterida $(2 \mathrm{mg} / \mathrm{dia})$. Também foram iniciadas aplicações mensais, subcutâneas com dipropionato de betametasona $(5 \mathrm{mg})$ + fosfato dissódico de betametaona $(2 \mathrm{mg})$, diluídos em $2 \mathrm{~mL}$ de lidocaína $(20 \mathrm{mg} / \mathrm{g})$, com a finalidade de estabilizar a área de calvície, no entanto a paciente parou o tratamento por contra própria.

A paciente retornou para uma nova consulta em junho de 2018 e afirmou que havia parado o tratamento que foi instituído anteriormente por conta própria e, consequentemente, constatou-se que houve um aumento da área da calvície. Assim, foi prescrito metilprednisolona subcutâneo que foi utilizado até junho de 2020. Em junho de 2019 associou a hidroxicloroquina $400 \mathrm{mg}$ com a metilprednisolona subcutânea, atualmente a paciente permanece utilizando hidroxicloroquina.

Em outubro de 2019, a paciente compareceu para a consulta de retorno e nessa ocasião foi dado início a tratamento com isotretinoína $20 \mathrm{mg}$ por dia. A utilização do referido medicamento foi finalizada em dezembro de 2020, pelo que se constatou que o seu uso teve como resposta a estabilização da alopecia. Ao questionar a paciente sobre quais as perspectivas quanto ao tratamento, a mesma disse que almejava que tivesse ocorrido a repilação, pois isso interferia na sua autoestima. 


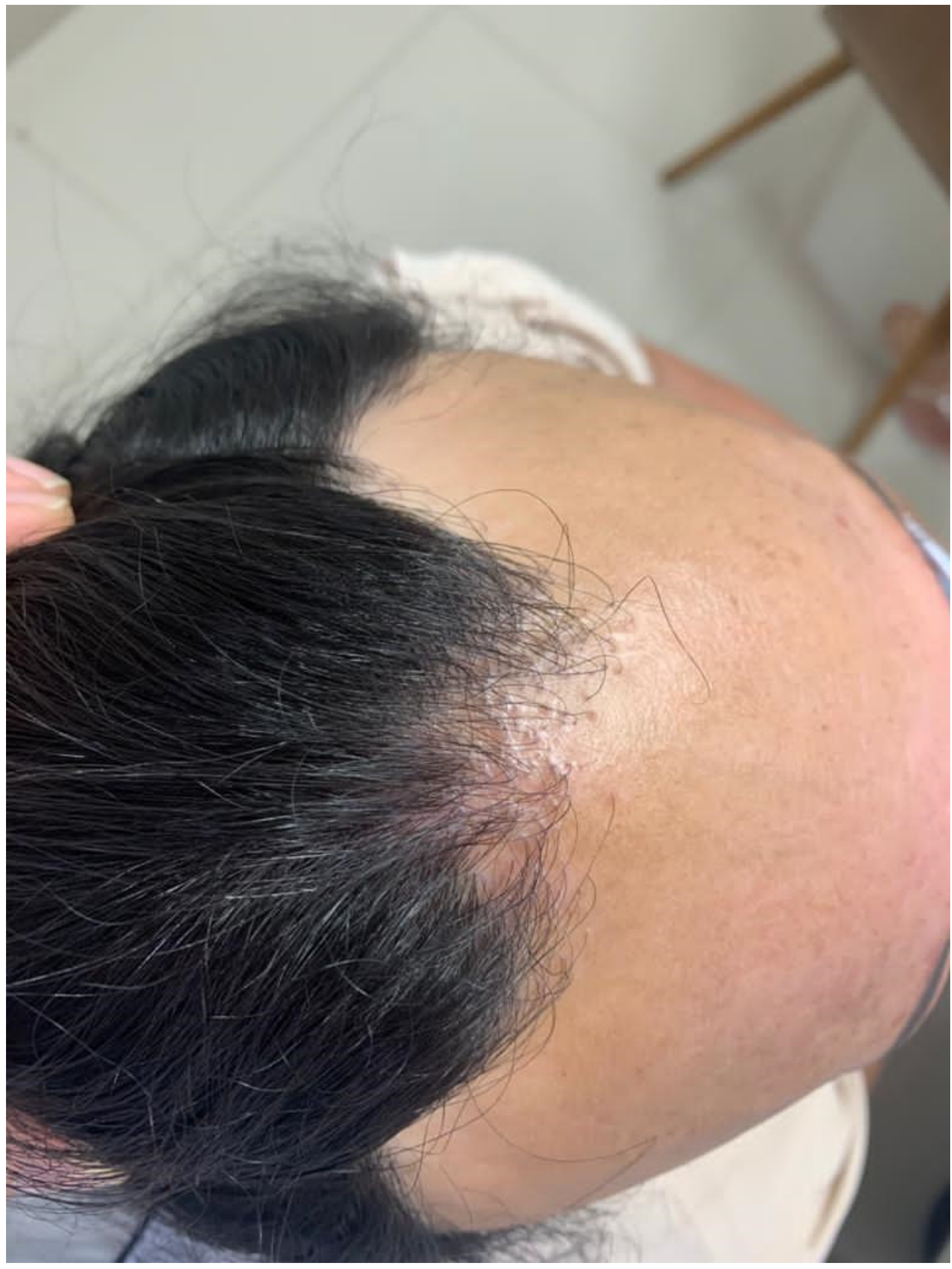

Figura 1. Aspecto clínico da lesão em região frontal.

Fonte: Autoria própria. 


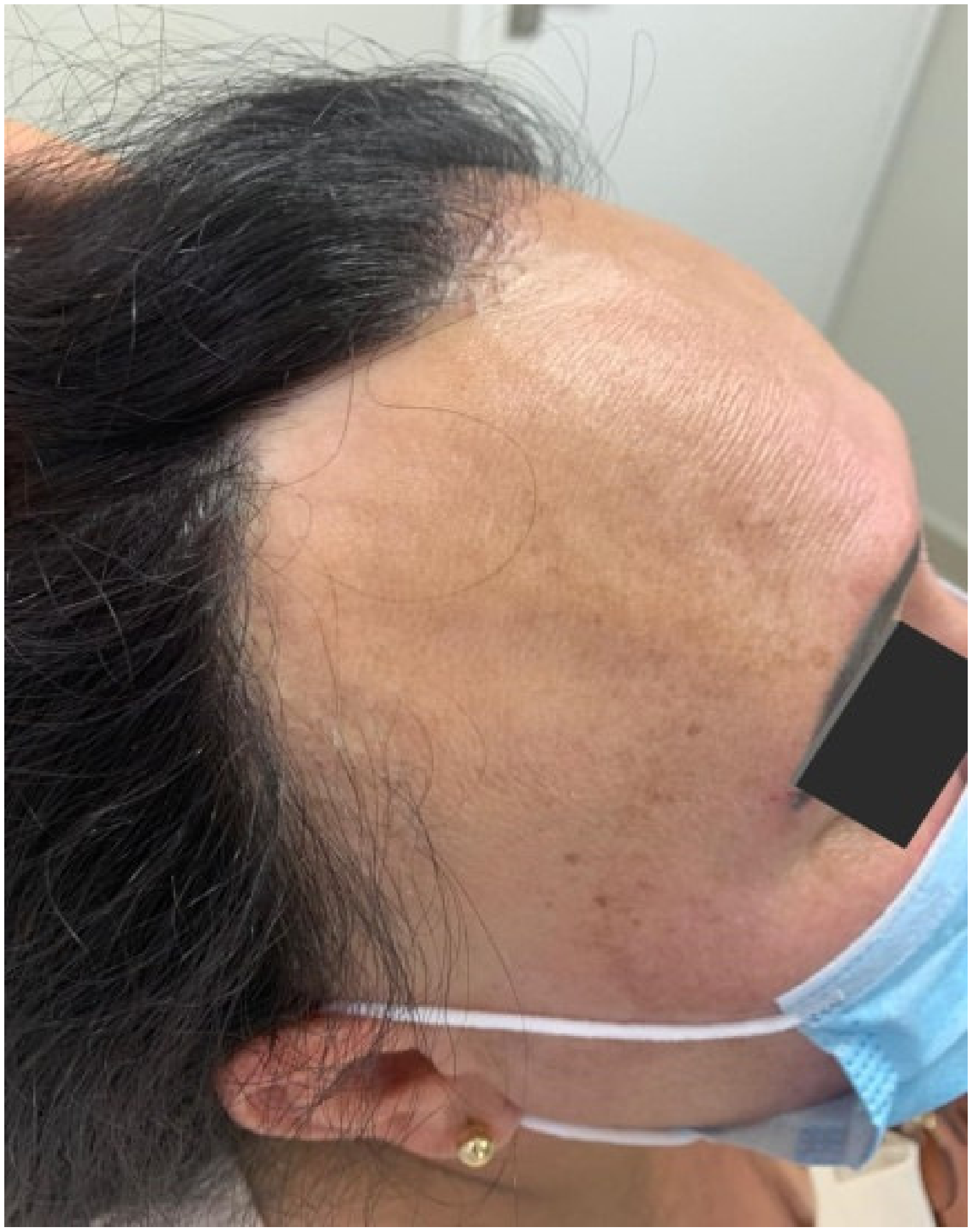

Figura 2. Aspecto clínico da lesão em região temporoparietal.

Fonte: Autoria própria. 


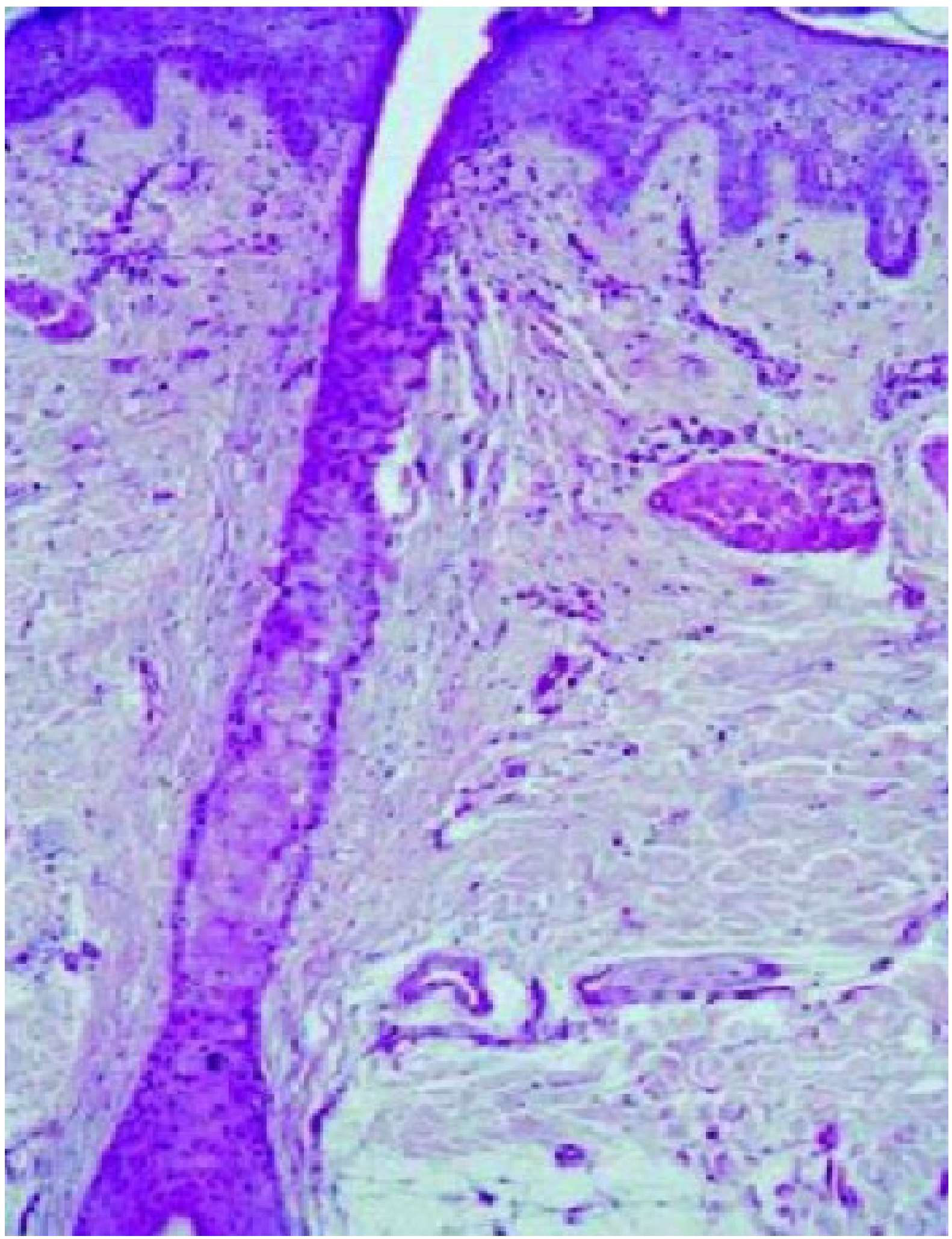

Figura 3. Aspecto clínico de lesão corado pelo método HE, evidenciando fibrose de folículo piloso e infiltrado linfocítico, circundando o folículo piloso.

Fonte: Autoria própria. 


\section{Paciente 2}

A Paciente J.D. do gênero feminino, 60 anos, parda, viúva, com segundo grau completo, aposentada, residente e procedente da cidade de São José do Egito-PE, em 22/11/2020, compareceu ao Centro de Saúde Frei Damião relatando início de madarose, há mais ou menos dez anos e, subsequentemente, apresentando alopecia, principalmente, na região frontal, considerando que o início da alopecia pode estar atrelado a momentos de estresse.

Negou outras queixas, comorbidades, alergias a medicações, etilismo, tabagismo e que fazia uso de progesterona $20 \mathrm{mg}$ e estradiol $1 \mathrm{mg}$ por via intradérmica. Baseado em suas manifestações e características clínicas da alopecia, foi levantada a hipótese diagnóstica de alopecia fibrosante frontal. Dessa forma, foi prescrito isotretinoína $20 \mathrm{mg}$ por dia e solução capilar composta por Minoxidil $5 \%$, estradiol $0,025 \%$, VEGF $1 \%$ e BFGF $1 \%$ que deve ser aplicada diariamente à noite no couro cabeludo.

A paciente retornou após dois meses e relatou que havia percebido melhora significativa no quadro de alopecia, mas queixou-se de ressecamento das mucosas, pelo que, diante dessa reação adversa houve uma mudança na posologia da medicação, sendo utilizada $20 \mathrm{mg}$ de isotretinoína por dia, durante cinco dias, sendo feita uma pausa de dois dias. Além disso, foram solicitados exames laboratoriais, entre eles: transaminase oxalacética, transaminase pirúvica, ureia, creatinina, hemograma, triglicerídeos, glicemia de jejum, gama glutamil transferase, os quais apresentaram resultados dentro da normalidade. Ao questionar a paciente sobre quais as perspectivas quanto ao tratamento, a mesma disse almejar que ocorresse a repilação, uma vez que isso interfere na sua autoestima. 


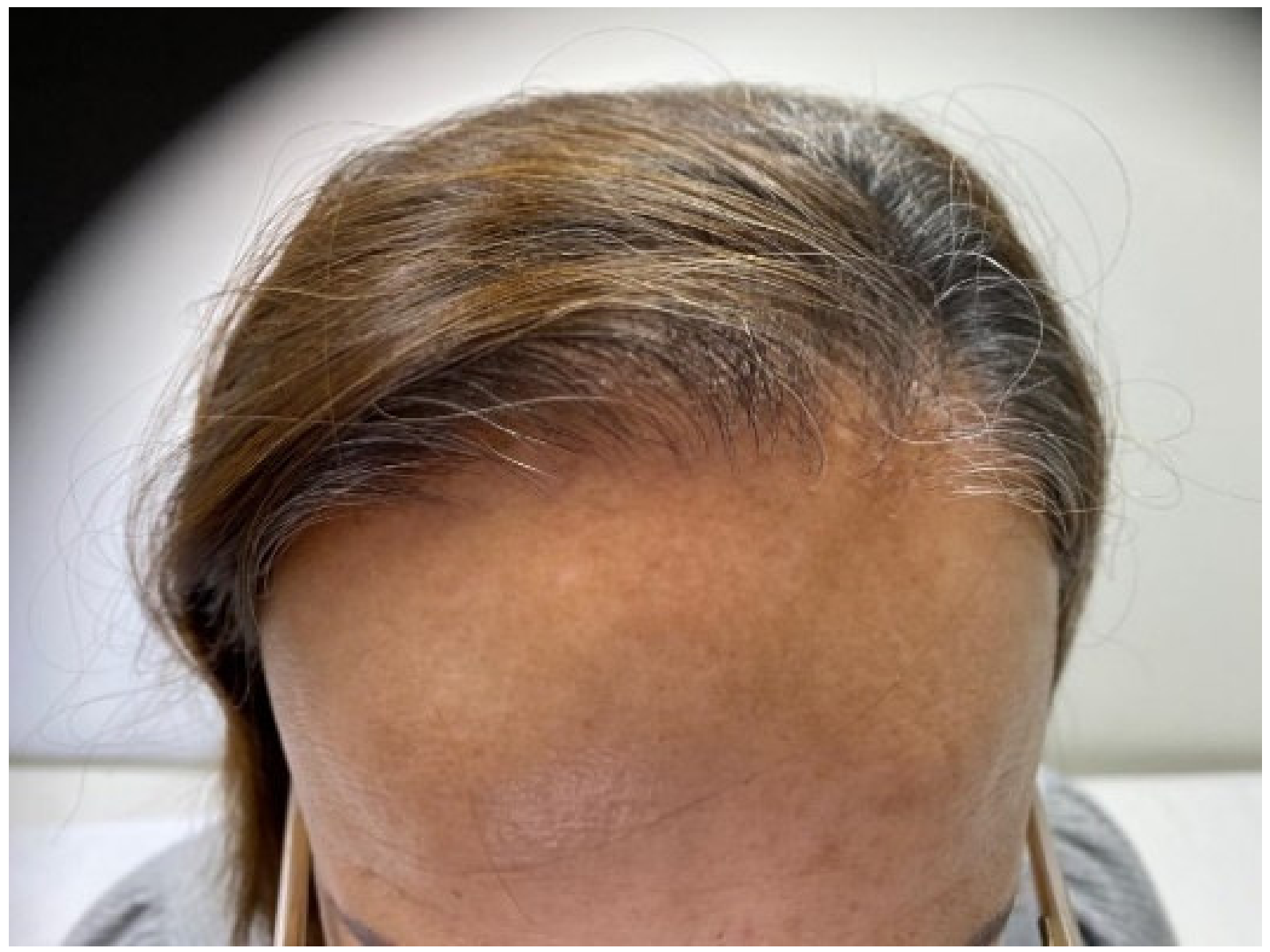

Figura 4. Aspecto clínico da lesão em região frontal.

Fonte: Autoria própria. 


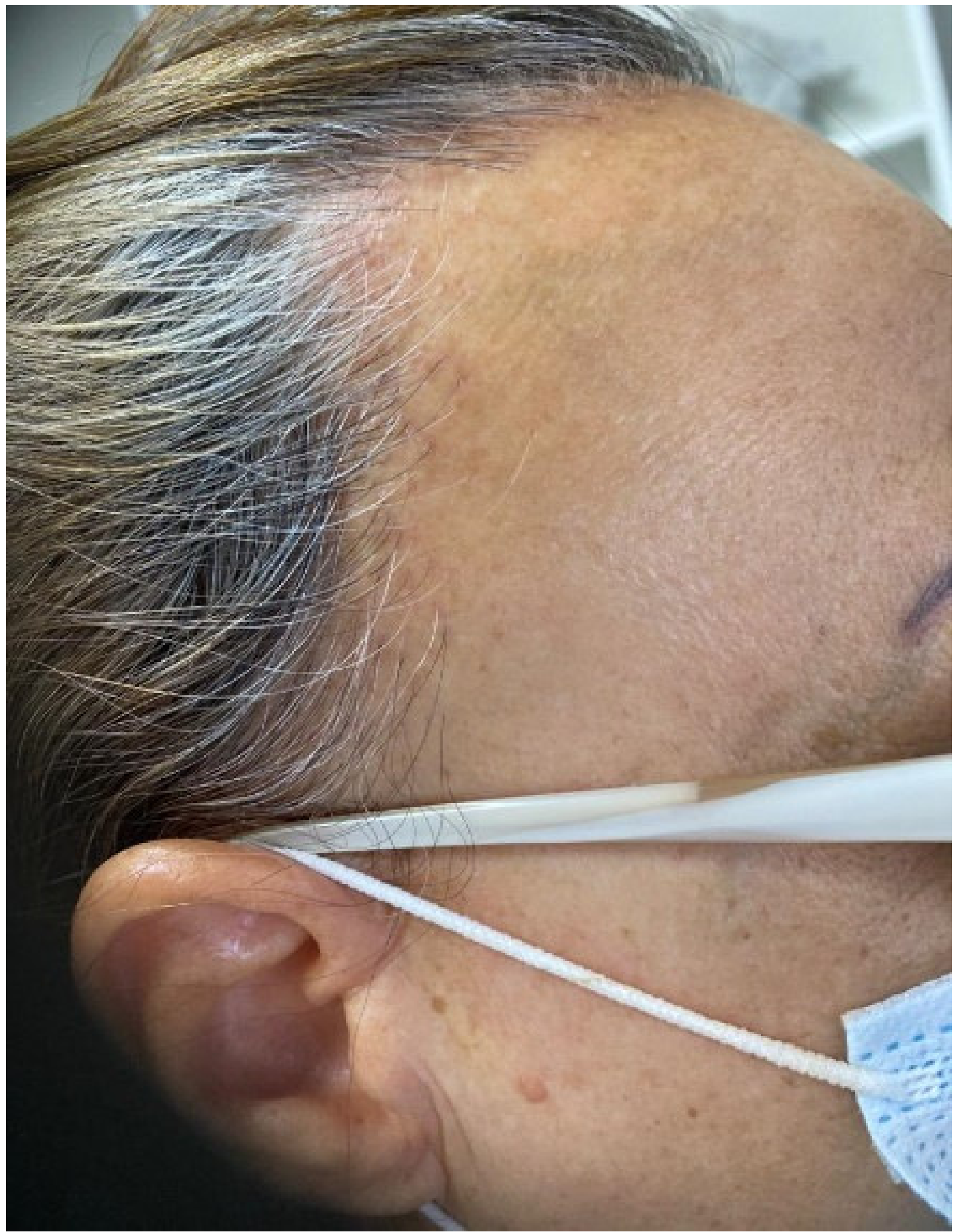

Figura 5. Aspecto clínico da lesão em região temporoparietal.

Fonte: Autoria própria. 


\section{DISCUSSÃO}

A alopecia fibrosante frontal (AFF) foi inicialmente relatada em 1994 por Kossard, na Austrália, em mulheres que se encontravam no período da pósmenopausa (MUINARI-BRENNER et al., 2007). É considerada uma alopecia cicatricial linfocítica primária de curso crônico. O padrão mais comum de acometimento observado é a perda de cabelo na região frontotemporal e nas sobrancelhas. Atualmente, não se sabe ao certo qual a patogênese envolvida na AFF, sendo descrita várias hipóteses para tal doença. Além disso, não existem tratamentos validados ou aprovados para essa patologia (LORIZZO; TOSTI, 2019).

Segundo Tosti e Piraccini (2014), a AFF foi relatada inicialmente por Kossard em 1994, sendo que a maior parte dos casos descritos na literatura acometeram uma maior parcela de mulheres brancas no período pós-menopausa, com menor frequência pode surgir em mulheres na pré-menopausa. É uma condição rara em homens e pessoas da raça preta e asiática.

Levando em consideração o estudo de Vãnó-Galvan et al. (2014), o perfil da população acometida pela alopecia fibrosante frontal apresentou algumas modificações quando comparada a relatos anteriores, continua acometendo em maior percentual mulheres, no entanto são mulheres mais jovens e antes da menopausa. A paciente mais jovem descrita na literatura tinha 18 anos quando apresentou as manifestações clínicas da AFF (BANKA et al., 2014).

Segundo Jorge (2018), existe uma predominância em pacientes caucasianos, levando em consideração que a etnia apresenta variações de acordo com o país em que foi realizado o estudo, como grande parte dos estudos foi descrita na Austrália, Europa e na América do Norte, assim há uma predominância de pacientes caucasianos.

A patogênese da AFF ainda não foi elucidada, no entanto existem várias teorias sobre fatores que podem ser desencadeantes, como a ação hormonal, a inflamação neurogênica, o tabagismo, os filtros ultravioletas (UV) e ingredientes de produtos para rosto sem enxágue, além dessas pressuposições, uma base genética 
também foi relatada, pois a AFF pode se mostrar presente em membros de uma mesma família (LORIZZO; TOSTI, 2019).

Em 1994, Kossard relata a hipótese da influência de fatores hormonais na AFF, posteriormente, diversas pesquisas reafirmam essa ideia e relataram ainda uma maior exclusividade de acometimento do sexo feminino (VAÑÓ-GALVÁN et al., 2014), além de ser mais prevalente em mulheres no período pós menopausa (MACDONALD et al., 2012; VAÑÓ-GALVÁN et al., 2014) e na ação terapêutica de terapia medicamentosa anti-androgênica (TOSTI et al., 2005; GEORGALA et al., 2009; LANDIZUNSKI et al., 2013).

Mesmo havendo muitos argumentos, não se sabe ao certo qual o hormônio específico que ocasiona a doença. Foi exposto por Tosti et al. (2005) e Landizunski et al. (2013) que por acometer áreas androgênio dependentes e a existência de uma resposta a uma medicação anti-androgênica, sendo está uma possibilidade de explicar a influência hormonal no surgimento dos quadros de AFF.

Além disso, em 2016, foi especulado, pela primeira vez, por Aldoori et al. (2016), o estudo sobre a utilização de protetores solares na patogênese da AFF. A fim de investigar melhor a teoria, de que existe relação entre a patogênese da AFF e a utilização de cremes faciais, foram realizados outros estudos, os quais foram encontrados resultados que mostraram uma diferença relevante no maior uso de cremes faciais e protetores solares em pacientes com AFF (DONATI, A., 2011; GAVIOLI et al.,2015; KIDAMBI et al.,2016). Por fim, é importante ressaltar que mesmo havendo tantas explicações nenhuma delas consegue dizer ao certo o que ocorre na patogênese da AFF e até então nenhuma patogênese foi elucidada.

A manifestação clínica mais comum é a recessão da linha frontotemporal do couro cabeludo, essa recessão se manifesta de forma simétrica e bilateral. A pele da região acometida pela alopecia vai apresentar-se lisa, com perda de orifícios e pálida, nessa região se forma uma cicatriz de caráter irreversível. Além disso, os portadores de AFF em 20 a 35\% dos casos podem queixar-se de coceira e até mesmo dor na região da alopecia. Pode ocorrer o envolvimento das sobrancelhas, e é observado, em cerca de $95 \%$ dos casos, que o padrão de acometimento é variável, sendo o mais comum a queda de cabelo na região lateral que tem o 
potencial de evoluir para perda total ou quase total da sobrancelha (ESTEBANLUCÍA; MOLINA-RUIZ; REQUENA, 2017).

Alguns sinais e sintomas são associadas a estágios mais graves da doença, tais como: perda de cabelos em regiões de articulação, corpo, cílios e presença de pápulas faciais. Por fim, existem achados clínicos que foram relatadas na literatura recentemente, dentre elas: pontos vermelhos glabelares, depressão das veias frontais e máculas pigmentadas faciais (ESTEBAN-LUCÍA; MOLINA-RUIZ; REQUENA,2017).

Destaque-se que o diagnóstico da alopecia fibrosante frontal é fácil de ser estabelecido, pois essa patologia apresenta um quadro clínico bem padronizado. Entretanto, em estágios iniciais pode gerar uma dificuldade quando relacionado aos diagnósticos diferenciais de outros tipos de alopecia. Então, pode ser lançado mão de outras técnicas diagnósticas complementares, dentre elas: tricoscopia ou histopatológico (ESTEBAN-LUCÍA; MOLINA-RUIZ; REQUENA, 2017).

Segundo Moreno-Arrones et al. (2017), o prognóstico da alopecia fibrosante frontal é influenciado pelo padrão clínico em que a pessoa acometida por AFF se encontra, existem três padrões clínicos em que o portador da patologia pode se enquadrar e esses apresentam diferentes prognósticos. As pessoas que são classificadas como padrão III tem maior predisposição a progredir de forma mais lenta, e tem menor envolvimento das sobrancelhas, quem apresenta o padrão II (difuso) tem o pior prognóstico.

O tratamento da AFF ainda é alvo de pesquisas, pois ainda não existe um tratamento validado ou aprovado. A terapia medicamentosa geralmente prescrita inclui medicações tópicas como: minoxidil, corticosteróides e inibidores da calcineurina, também podem ser utilizadas medicações com ações sistêmica como: $5 \alpha$-redutase, hidroxicloroquina e retinóides. Além disso, o acetonido de triancinolona intralesional pode ser usado como terapia medicamentosa voltada para as sobrancelhas. Outras medicações que também podem ser utilizadas são: pioglitazona, naltrexona, tofacitinibe e lasers (LORIZZO; TOSTI, 2019).

A terapia medicamentosa é prolongada, pois a AFF apresenta caráter crônico, a falta de ensaios clínicos randomizados faz com que não se saiba ao certo a eficácia dos tratamentos disponíveis. A escolha das medicações é influenciada por 
fatores como:localização da alopecia, estágio da doença, inflamação e coceira. O recurso terapêutico pode ser modificado, tendo como principal parâmetro para essa mudança a resposta do paciente a medicação prescrita (LORIZZO; TOSTI, 2019).

\section{CONSIDERAÇÕES FINAIS}

As pacientes retratadas nos casos clínicos apresentam alopecia fibrosante frontal que foram diagnosticadas anos após o surgimento dos primeiros sintomas, sendo a madarose o sintoma inicial mais prevalente. As pacientes foram tratadas com Isotretinoína oral e a medicação se mostrou eficaz, pois ocorreu a estabilização da alopecia. A perda capilar gera impacto na feminilidade dessas mulheres, sendo comum a relação da alopecia com baixa autoestima.

A escassez de estudos sobre tal patologia e o pouco conhecimento sobre sua etiologia faz com que o diagnóstico seja dado tardiamente, pois os sintomas apresentados inicialmente podem induzir a diagnósticos diferenciais de outras alopecias. Devido ao número reduzido de Ensaios Clínicos Randomizados dificulta em saber ao certo a eficácia dos tratamentos disponíveis.

\section{REFERÊNCIAS BIBLIOGRÁFICAS}

ALDOORI, N. et al. Frontal fibrosing alopecia: possible association with leave-on facial skin care products and sunscreens; a questionnaire study. British Journal of Dermatology, v. 175, n. 4 , p. $762-767,2016$.

BANKA, N. et al. Frontal fibrosing alopecia: a retrospective clinical review of 62 patients with treatment outcome and long-term follow-up. International journal of dermatology, v. 53, n. 11, p. 1324-1330, 2014.

DONATI, A. et al. Facial papules in frontal fibrosing alopecia: evidence of vellus follicle involvement. Archives of dermatology, v. 147, n. 12, p. 1424-1427, 2011.

ESTEBAN-LUCÍA, L.; MOLINA-RUIZ, A. M.; REQUENA, L. Actualización en alopecia frontal fibrosante. Actas Dermo-Sifiliográficas, v. 108, n. 4, p. 293-304, 2017.

GAVIOLI, CFB. et al. Frontal fibrosing alopecia etiology: Do environmental and behavioral factors play a role? In: Ninth World Congress of Hair Research. Miami, Florida. 2015. p. 18- 
21.

GEORGALA, S. et al. Treatment of post-menopausal frontal fibrosing alopecia with oral dutasteride. Journal of the American Academy of Dermatology, v. 61, n. 1, p. 157-158, 2009.

JORGE, ARCD. Caracterização clínica e laboratorial do acometimento dos folículos velos e da epiderme da face, pescoço e região anterossuperior do tórax na alopecia frontal fibrosante. Tese de Doutorado. Universidade de São Paulo.

$\mathrm{KIDAMBI}, \mathrm{AD}$. et al. Frontal fibrosing alopecia in men: an association with leave-on facial cosmetics and sunscreens. British Journal of Dermatology, v. 175, p. 46-46, 2017.

KIENLE, Gunver S.; KIENE, Helmut. Como escrever um relato de caso. Arte Médica Ampliada, v. 31, n. 2, p. 34-7, 2011.

KOSSARD, S. Postmenopausal frontal fibrosing alopecia: scarring alopecia in a pattern distribution. Archives of dermatology, v. 130, n. 6, p. 770-774, 1994.

LADIZINSKI, B. et al. Alopecia frontal com fibrose: uma revisão retrospectiva de 19 pacientes atendidos na Duke University. Jornal da Academia Americana de Dermatologia, v. 68, n. 5, pág. 749-755, 2013.

LORIZZO, M.; TOSTI, A. Frontal fibrosing alopecia: an update on pathogenesis, diagnosis, and treatment. American Journal of Clinical Dermatology, p. 1-12, 2019.

MACDONALD, A.; CLARK, C.; HOLMES, S. Frontal fibrosing alopecia: a review of 60 cases. Journal of the American Academy of Dermatology, v. 67, n. 5, p. 955-961, 2012.

MORENO-ARRONES, OM. et al. Frontal fibrosing alopecia: clinical and prognostic classification. Journal of the European Academy of Dermatology and Venereology, v. 31, n. 10, p. 1739-1745, 2017.

MULINARI-BRENNER, F. et al. Alopecia frontal fibrosante: relato de seis casos Frontal fibrosing alopecia: report of six cases. Anais Brasileiros de Dermatologia, v. 82, n. 5, p. 439-44, 2007.

PARENTE, RAPHAEL CÂMARA MEDEIROS; OLIVEIRA, M. A. P.; CELESTE, ROGER KELLER. Relatos e série de casos na era da medicina baseada em evidência. Brazilian Journal of Videoendoscopic Surgery, v. 3, n. 2, p. 67-70, 2010.

RAKOWSKA, A. et al. Eficácia da isotretinoína e acitretina no tratamento da alopecia fibrosante frontal: análise retrospectiva de 54 casos. Jornal de drogas em dermatologia: JDD, v. 16, n. 10, pág. 988, 2017.

SMIDARLE, DN.; SEIDL, M.; SILVA, RCD. Frontal fibrosing alopecia: case report. Anais brasileiros de dermatologia, v. 85, n. 6, p. 879-882, 2010.

TOSTI, A. et al. Alopecia fibrosante frontal em mulheres na pós-menopausa. Jornal da Academia Americana de Dermatologia, v. 52, n. 1, pág. 55-60, 2005.

TOSTI A.; PIRACCINI BM. Alopecia fibrosante frontale. Tricologia ambulatoriale. Springer, Milano. 2016.

VAÑÓ-GALVÁN, S. et al. Frontal fibrosing alopecia: a multicenter review of 355 patients. Journal of the American Academy of Dermatology, v. 70, n. 4, p. 670-678, 2014. 